\title{
Optimization Deployment Strategy of Mine Roadway Wireless Sensor Network
}

\author{
Bin $\mathrm{Wu}^{1}$, Jian Luo ${ }^{1}$ and Lingbing Tang ${ }^{2, *}$ \\ ${ }^{1}$ Department of Automation, Xiamen University, Xiamen, Fujian, 361005, China; ${ }^{2}$ School of Computer and Information \\ Engineering, Hunan University of Commerce, Changsha, Hunan, 410205, China
}

\begin{abstract}
Further research has been done in order to reduce the energy consumption of the mine roadway wireless sensor nodes and prolong the lifetime of the network. On the basis of analysis and study on the existing linear wireless sensor network nodes deployment, the node distance optimization deployment scheme with sinks in the center is proposed. The calculation method for optimized distance between nodes is provided. The experimental simulation prove that, compared to the traditional deployment scheme, this scheme can effectively solve the problem of "energy hole", greatly reduce the energy consumption of single sensor node and whole system, and prolong the network life cycle. For mine roadway with long distance, the optimization deployment scheme with multisinks shall be used. According to number of optimized sinks, the monitoring area in the mine roadway is divided, the total energy consumption of system is minimized, the energy consumption of sensor nodes is balanced, and the network life cycle is significantly lengthened.
\end{abstract}

Keywords: Distance optimization, mine roadway, multisinks, node deployment, WSN.

\section{INTRODUCTION}

Safe mining in the underground coal mine is an important guarantee of coal industry development in a healthy and ordered way. The underground mining environment for coal resources is complex and varied, and the parameters of surrounding rocks in mine roadway including engineering mechanical structure, hydrology, ground pressure and air are constantly changing. When one parameter reaches a certain degree, mine disasters such as gas explosion, water inrush, gas poisoning and fire may be caused. Therefore, mine safety environmental monitoring is an important and essential part during coal mining $[1,2]$. Since the mine roadway is narrow and long in poor environmental conditions, the traditional wired detection equipment is not easy to install and even very dangerous. With the development of sensor and wireless communication, Wireless Sensor Networks (WSN) are widely used in areas such as mine safety monitoring for the sensor node featuring small volume, low cost, low consumption and self-organization [3]. Through sensor nodes deployed in the monitoring area, WSN realizes real-time monitoring of parameters such as temperature and humidity, gas density, carbon monoxide concentration, equipment conditions and process control. In this way, the monitoring level of coal mine safety production is effectively improved and the accident potential is reduced [4]. One hot spot of WSN study is node deployment, which aims to solve problems such as number and location of nodes required in the working area, determines the effect of physical space monitored by the sensor, and further impacts the network service quality, thus is closely related to the actual applications. Due to

*Address correspondence to this author at the School of Computer and Information Engineering, Hunan University of Commerce, Changsha, Hunan, 410205, China; E-mail: lingbingtang@gmail.com the topographical characteristics of underworkings, the wireless sensor nodes can be only deployed along the working area, determines the effect of physical space narrow and long roadway, so WSN is a linear topological structure network. Multiple hop transmission is usually used for communication between the node and base station, the node is closer to the sink, more data are required to be transferred, and the energy consumption is higher, so "energy hole" can be caused due to premature death of node close to the sink, thus greatly reducing the network life cycle $[5,6]$. Reasonable deployment of wireless sensor nodes can reduce the energy consumption and prolong the network life cycle. Study on mine roadway WSN deployment has reference value in the study on WSN approximate to linear structure of tunnel, bridge and subway.

At present, some achievements have been gained in node deployment study for linear WSN. Lu Kezhong et al. proposed a node deployment scheme for linear WSN based on the density [7], which provides a density formula for sensor node deployment, to make the ratio of total energy and energy consumption speed in all areas, balanced, thus solving the problem of "energy hole". Tian Feng et al. proposed k coverage WSN deployment strategy [8], which uses the coverage in areas of isosceles triangle and node grouping, solving the $\mathrm{k}$ coverage and fault tolerance of WSN under the linear environment of mine. Ma Hongfeng et al. proposed a clustering linear topological structure based on WSN [9], which reduces data communication traffic, thus saving network energy to a certain extent. Zhang and Hou proposed the optimized complete cover arithmetic (OGDC), and Wang proposed the cover degree configuration protocol (CCP), which are both designed on the condition that random deployment of nodes is carried out in an enough dense manner [10]. Zhang Ruihua et al. proposed an unequal distance deployment method based on the energy efficient chained WSN 
nodes [11], which solves the problem that sensor node close to sink may "die" early, thus balancing the energy consumption of intermediate nodes and maximize the network life cycle. However, this method is applicable only to network with short distance, for chained WSN with long distance, as the hop count increases, the network life cycle is significantly reduced [12]. In short, the deployment strategies above are not suitable for the mine roadway with complex environment.

In this paper, a WSN deployment strategy suitable for the mine roadway is provided. It proposes that the sinks are located in the middle of network line, the sensor nodes are deployed on both sides of the mine roadway with unequal distance, so with the center of sinks, the multiple hop transmission information on both sides is sent to the sinks, thus maximizing the network life cycle and reducing the node energy consumption.

\section{SYSTEM MODEL}

According to the spatial location and shape, the mine roadway can be divided into vertical, horizontal and inclined roadway. This paper focuses on the narrow and long horizontal roadway by assuming the chain area is linear, this method is also applicable to the vertical and inclined roadway..

The research shows that the wireless sensor node energy consumption mainly lies in data sending and receiving [13]. For easy analysis, this paper ignores the other energy consumption. For this, the energy consumption model used in this paper is as follows [14]:

$$
\begin{aligned}
& E_{t}=b E_{\text {elec }}+b \xi d^{n} \\
& E_{r}=b E_{\text {elec }}
\end{aligned}
$$

Where the energy consumption for data sending and receiving is respectively $E_{t}$ and $E_{r} . b$ refers to the amount of information perceived in unit time, $E_{\text {elec }}$ refers to energy consumption for sending and receiving unit bit data by node radio-frequency circuit, $\xi$ refers to a constant related to the path loss exponent, $\mathrm{n}$ refers to the path loss exponent, generally being 2 , and $d$ refers to the transmission distance between the sending node and receiving node.

In order to analyze data flow in each area of the linear monitoring area, this paper first makes the following assumptions:

1) Sensor nodes are exactly the same, with the same initial energy, transmission distance and energy consumption model, and the power can be adjusted according to different transmission distance;

2) The sink has enough energy, the position is fixed, and its energy consumption is not taken into consideration;

3) The determined deployment method is used for the sensor, and the precise location can be given for installation;

4) Multiple hop transmission is usually used for communication between the sensor node and sink, and the most distance between sensor node and sink is greater than the wireless communication distance of sensor nodes.
5) The monitoring scope of all sensor nodes covers the whole monitoring area.

\section{SENSOR NODE DEPLOYMENT METHOD}

At present, most of the research models of linear wireless sensor network node are meant to locate the sink at one end of the network link, so the sensor nodes are transmitted to the sink via multihop method, causing the sensor node closest to the sink premature death and network break off [15]. That's why this paper, based on the existing research, proposes an improved model structure locating the sink in the middle of the link to carry out node distance optimization deployment with sink as the center to both ends (recorded as OD1). Meanwhile, due to the movement of personnel or equipment in the mine, the random deployment method will not only waste nodes but also easily cause manmade damage. By using the fixed deployment method, the nodes are deployed on both sides of the roadway in an ordered way, thus avoiding damage to the nodes.

The mine roadway sensor node optimization deployment model used in this paper is as shown in Fig. (1).

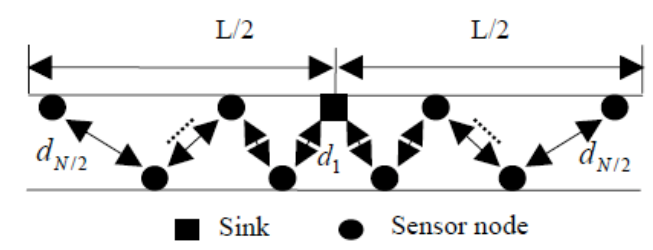

Fig. (1). OD1 optimization deployment model.

The sensor nodes are deployed on both sides of sink in a non-uniform symmetry along the narrow and long roadway. Assuming the roadway length is $\mathrm{L}$, the total number of sensor nodes is $\mathrm{N}$, the distance between nodes is successively $d_{1}, d_{2}, \ldots, d_{N / 2}$.

Since the model is symmetrical to sink, during analysis of node energy consumption, only one half is enough. Assuming the node $\mathrm{NO}$ is successively $s_{1}, s_{2}, \ldots, s_{N / 2}, E_{i}$ refers to the energy consumption of the ith node in unit time.

The node $s_{i}$ is responsible for the transfer of data package detected by the node $s_{i+1} \sim s_{N / 2}$. According to the energy consumption model (1), the energy consumption for receiving $\mathrm{N} / 2-\mathrm{i}$ data package from peripheral nodes is:

$E_{i_{-} r}=\left(\frac{N}{2}-i\right) b E_{\text {elec }}, 1 \leq i \leq \frac{N}{2}$

The energy consumption for the node $s_{i}$ transmitting the data package of itself and peripheral nodes to the node $s_{i-1}$ :

$E_{i_{-} t}=\left(\frac{N}{2}-i+1\right) b\left(E_{\text {elec }}+\xi d_{i}^{2}\right), 1 \leq i \leq \frac{N}{2}$

Therefore, the total energy consumption of the node $s_{i}$ :

$E_{i}=(N-2 i+1) b E_{\text {elec }}+(N / 2-i+1) b \xi d_{i}^{2}$,

$1 \leq i \leq N / 2$

From the formula (4), the smaller the value $i$ is, the node is closer to sink, and the energy consumption is greater.

The total energy consumption of the system: 


$$
E=2 \sum_{i=1}^{N / 2} E_{i}=N^{2} b E_{\text {elec }} / 2+2 b \xi \sum_{i=1}^{N / 2}(N / 2-i+1) d_{i}^{2}
$$

In fact, the length of roadway can be several hundreds and even thousands meters, but the width is less than ten meters, so it is approximate to

$$
\sum_{i=1}^{N / 2} d_{i}=L / 2
$$

Since $N, L$ are fixed values, the total energy consumption of the system $E$ has a minimum value in the finite interval, corresponding to a group of optimal value $d_{i}$. The partial derivative is determined by the formula (5), and the distance from each node to the adjacent two sensor nodes with balanced energy consumption is taken into consideration:

$d_{i}=L / 2 /(N / 2-i+1) \sum_{i=1}^{N / 2} 1 / i$

The formula (6) is substituted into the formula (5), the total energy consumption of the system is obtained:

$$
E=N^{2} / 2 b E_{\text {elec }}+b \xi L^{2} / 2\left(\sum_{i=1}^{N / 2} 1 / i\right)^{-1}
$$

When $i=N / 2, s_{i}$ refers to the end node farthest to sink, with only energy consumption for sending data but not receiving data, and the energy consumption is the lowest:

$$
E_{N / 2}=E_{N / 2 \_t}=b E_{\text {elec }}+\frac{1}{4} b L^{2} \xi\left(\sum_{i=1}^{N / 2} \frac{1}{i}\right)^{-1}
$$

When $i=1, s_{i}$ refers to the most front-end node closest to sink, and the energy consumption is the highest:

$$
E_{1}=E_{1_{-} t}+E_{1_{-} r}=(N-1) b E_{\text {elec }}+\frac{1}{2 N} b L^{2} \xi\left(\sum_{i=1}^{N / 2} \frac{1}{i}\right)^{-1}
$$

The comparison between the OD1 model and other two deployment models is as follows.

(1) The model for sink distance optimization deployment at one end of the roadway (recorded as OD2) is as shown in Fig. (2).

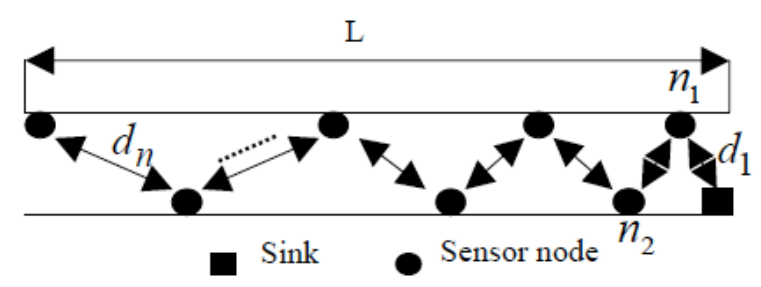

Fig. (2). OD2 optimization deployment model.

By analysis of the node energy consumption, the model in formula (1) can also be used, $p_{i}$ refers to the energy consumption of node $i$. Be reference to the analysis above, the node energy consumption can be determined by using the formula (2) and (3): $\left\{\begin{array}{l}p_{i_{-} r}=(N-i) b E_{\text {elec }} \\ p_{i_{-} t}=(N-i+1) b\left(E_{\text {elec }}+\xi d_{i}^{2}\right)\end{array}\right.$

So the energy consumption of $P_{i}$ is:

$P_{i}=(2 N-2 i+1) b E_{\text {elec }}+(N-i+1) b \xi d_{i}^{2}$,

$1 \leq i \leq N$

The total energy consumption of the system is:

$P=\sum_{i=1}^{N} P_{i}=N^{2} b E_{\text {elec }}+b \xi \sum_{i=1}^{N}(N-i+1) d_{i}^{2}, 1 \leq i \leq N$

Assuming $d_{1}+d_{2}+\cdots+d_{i}+\cdots+d_{N}=L$, the total energy consumption of the system $p$ has a minimum value in the finite interval, corresponding to a group of optimal value $d_{i}$. The partial derivative is determined by the formula (12), and the distance from each node to the adjacent two sensor nodes with balanced energy consumption is taken into consideration:

$$
d_{i}=\frac{L}{(N-i+1) \sum_{i=1}^{N} \frac{1}{i}}
$$

The formula (13) is substituted into the formula (12), the total energy consumption of the system is obtained:

$$
P=N^{2} b E_{\text {elec }}+b L^{2} \xi\left(\sum_{i=1}^{N} \frac{1}{i}\right)^{-1}
$$

By comparison of $E$ in the formula (7) and $P$ in the formula (14), it is obviously $E=P / 2$, that is, the total energy consumption of the system with optimization deployment scheme OD1 is half lower, and the energy consumption of each node is reduced. From this, the optimization deployment scheme OD1 is superior to OD2, greatly reducing the energy consumption of the system and prolonging the system life cycle.

(2) The model for sink uniformity optimization deployment in the middle (recorded as OD3) is as shown in Fig. (3).

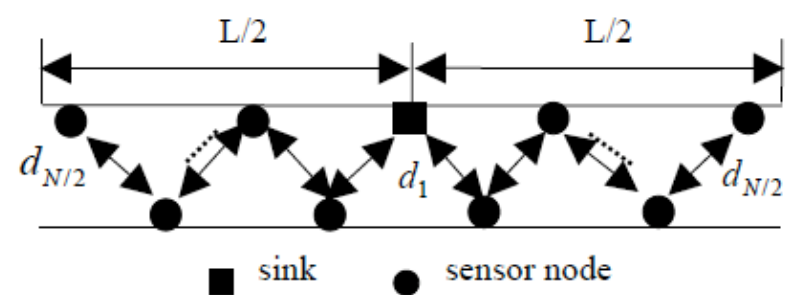

Fig. (3). OD3 optimization deployment model.

Since the sensor nodes are uniformly deployed on both sides of the roadway, $d_{i} \approx L / N$.Assuming that $T_{i}$ refers to the node energy consumption, the node energy consumption can be according to the formula (4):

$T_{i}=T_{i_{-} t}+T_{i_{-} r}=(N-2 i+1) b E_{\text {elec }}+\left(\frac{N}{2}-i+1\right) b \xi \frac{L^{2}}{N^{2}}$

The total energy consumption of the system: 


$$
T=2 \sum_{i=1}^{N / 2} T_{i}=\frac{N^{2}}{2} b E_{\text {elec }}+\frac{L^{2}}{2} b \xi\left(\frac{1}{N}+\frac{1}{2}\right)
$$

By comparison of formula (7) and formula (16), $T>E$, that is, the energy consumption of the system with optimization deployment OD3 is higher.

The life cycle of WSN in uniform deployment depends on the life cycle of the node closest to sink.

$T_{1}=(N-1) b E_{\text {elec }}+\frac{1}{2 N} b L^{2} \xi$

It is obvious that $T_{1}>E_{1}$, that is, the first node in optimization deployment OD3, will consume the energy earlier, so the network life cycle is shorter.

From two comparisons above, the total energy consumption of the system with optimization deployment OD1 is lower, and the energy consumption of single node is reduced, the node energy consumption is balanced, and the network life cycle is greatly prolonged.

\section{MULTISINK NODES OPTIMIZATION DEPLOY- MENT (RECORDED AS OD4)}

In fact, the mine roadway length can reach thousands meter or more, e.g., for roadway developing. Now, only single one sink is used for information processing, with the increase of node number, the hop count is increased during data transmission, and the energy consumption is increased, so the network life cycle is shortened.

Therefore, on the basis of the above study, this paper proposes one multisink node optimized structure, which divides the sensor nodes into several equal groups, each has a sink node deployed in the middle point, and the other nodes are deployed on both sides of the roadway with distance optimization, the model is as shown in Fig. (4). The increase of sink nodes will certainly greatly reduce the total energy consumption of the system.

Assuming there are $N$ sensor nodes in the monitoring area of roadway, the total length of the roadway is $L$, the monitoring area is divided into several sections with the same length $R$, and there are $m$ sensor nodes in each section.

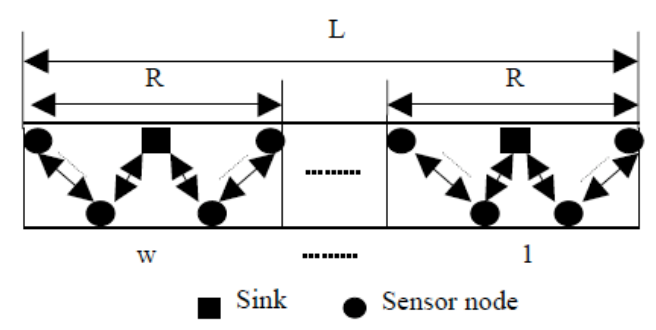

Fig. (4). OD4 optimization deployment model.

The nodes in each section send the information to the sink node via multi-hopping method, and the information transfer between sections is realized via the sink node.

Assuming that the roadway is divided into $w$ sections, $W_{i}(1 \leq i \leq w)$ refers to the energy consumption in the ith section.

$$
W_{i}=\frac{N^{2}}{2 w^{2}} b E_{\text {elec }}+\frac{1}{2 w^{2}} b \xi L^{2}\left(\sum_{j=1}^{N / 2 w} \frac{1}{j}\right)^{-1}, 1 \leq i \leq w
$$

The total energy consumption of the system:

$$
W=\sum_{i=1}^{w} W_{i}=\frac{N^{2}}{2 w} b E_{\text {elec }}+\frac{1}{2 w} b \xi L^{2}\left(\sum_{j=1}^{N / 2 w} \frac{1}{j}\right)^{-1}, 1 \leq i \leq w
$$

By comparison of formula (19) and formula (7), $W<E$, that is, the total energy consumption of the system is reduced as $w$ increases. However, the increase of sink node also make the equipment cost become higher.

Therefore, the sink node number and total energy consumption of network shall be taken into consideration of sensor node deployment optimization, that is, the minimum number sink nodes are required to minimize the energy consumption of the system.

Assuming the number of optimized sink nodes is $w_{\text {opt }}$, the maximum radius of sensor node communication is $R_{\max }$. Therefore, the problem of sink node network deployment optimization can be expressed as:

$$
\left\{\begin{array}{c}
\min W \\
\text { s.t. } \frac{L}{w_{\text {opt }}} \leq 2 R_{\max }
\end{array}\right.
$$

\section{SIMULATIONS}

To observe the factual influence of sensor node deployment in WSN on the energy consumption of the system, simulation experiments by using MATLAB to the optimization deployment OD1, OD2, OD3 and OD4 are carried out for contrastive analysis.

In the energy consumption model (1), $b=1024$ bit , Eelec $=50 \mathrm{~nJ} /$ bit , and $\xi=10 \mathrm{pJ} / \mathrm{bit}$.

(1) When the roadway length is fixed, with the increase of sensor node number, the comparison of energy consumption for optimization deployment OD1, OD2 and OD3 is as shown in Fig. (5). Assuming that the length of mine roadway $L=1000 \mathrm{~m}$, the total number of sensor nodes is increased from 1 to 150 .

From the Fig. (5), with the increase of sensor node number, the total energy consumption for three optimization deployments per unit of time is first decreased and then increased. The energy consumption for optimization deployments OD1 is obviously lower than the other two deployments, that is, the sink node optimization deployment in the middle greatly reduces the total energy consumption of the system and prolongs the life cycle.

(2) When the number of sensor nodes is fixed, with the increase of the roadway length, the comparison of energy consumption for optimization deployment OD1, OD2 and OD3 is as shown in Fig. (6). Assuming the total number of sensor nodes is $N=100$, the length of mine roadway $L$ is increased from $1 \mathrm{~m}$ to $1,000 \mathrm{~m}$.

From the figure above, with increase of length of mine roadway, the total energy consumption for three optimization 
deployments per unit of time is monotonically increased. However, the energy consumption for optimization deployments OD1 is obviously lower than the other two deployments, that is, the sink node optimization deployment in the middle greatly reduces the total energy consumption of the system and prolongs the life cycle.

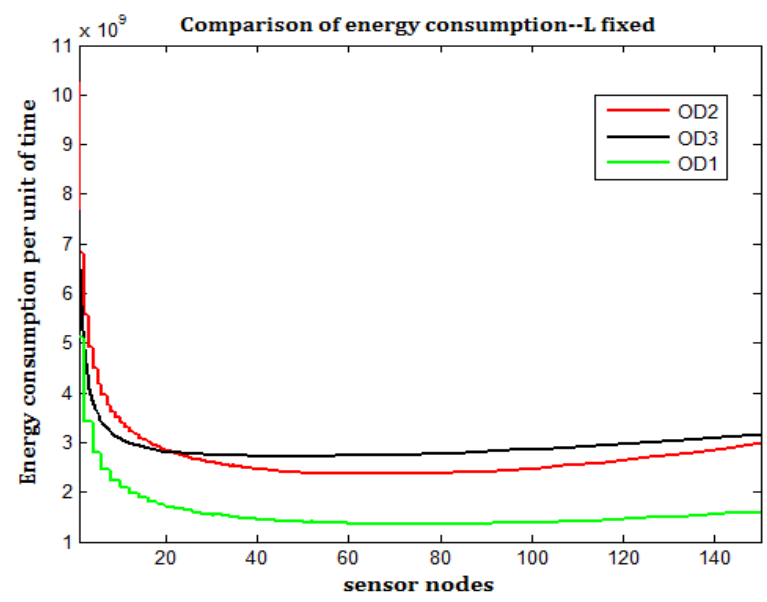

Fig. (5). Comparison of energy consumption for three optimization deployments with a fixed roadway length.

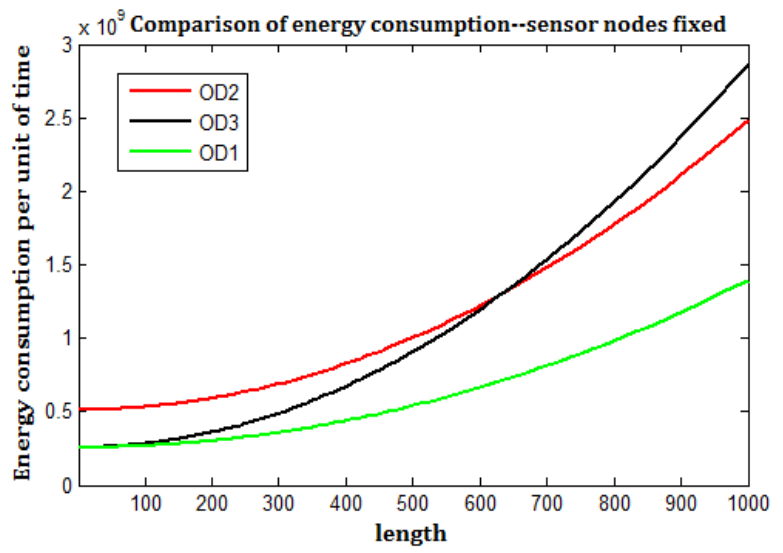

Fig. (6). Comparison of energy consumption for three optimization deployments with a fixed number of sensor nodes.

(3) The multisink nodes optimization deployment is used, it is assumed that $L=1000 m, N=100$. From Fig. (7), with the increase of sink nodes, the energy consumption of the system is decreased. When the number of sinks is greater than 10 , the decrease of energy consumption is not obvious. In consideration of the system equipment cost, the optimal number of sinks is 10 , that is, the roadway is divided into 10 areas with equal length, the length of each area is $100 \mathrm{~m}$, and the number of sensor nodes is 10 . On the basis of cost reduction, this scheme can minimize the network energy consumption and prolong the network life cycle to achieve the optimum application effects.

(4) The roadway length $L$ is fixed, the comparison of energy consumption for single sink and multisink deployment is as shown in Fig. (8). Assuming the roadway length $L=1000 \mathrm{~m}$, when the number of sensors is constantly in- creased, the comparison of energy consumption for two deployments is as shown in Fig. (8).

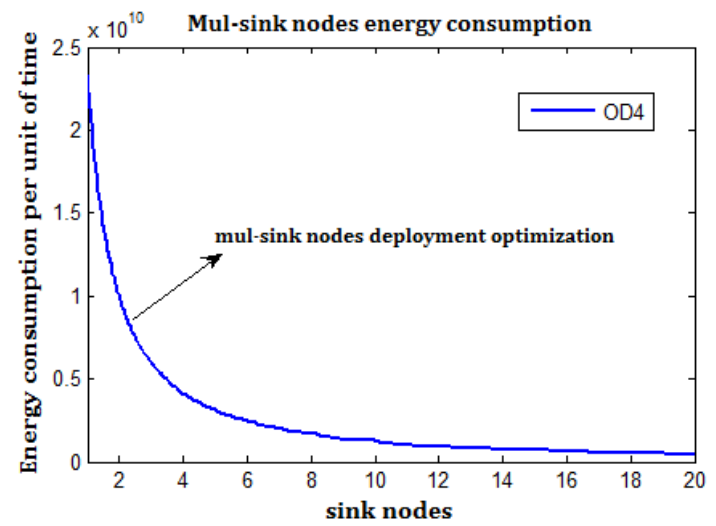

Fig. (7). Energy consumption for multisink nodes optimization deployment.

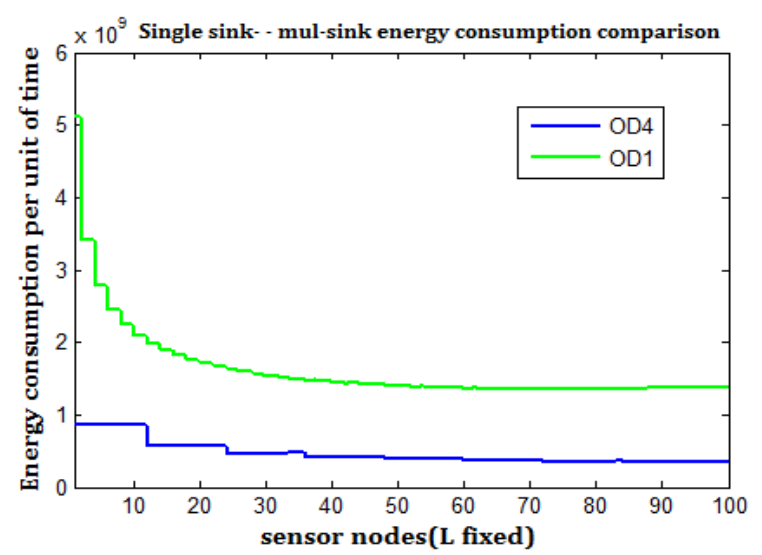

Fig. (8). Comparison of energy consumption for OD1 and OD4 with a fixed $\mathrm{L}$.

From the above figure, the energy consumption for mutisink optimization deployment is obviously decreased, and the network life cycle is greatly prolonged.

(5) When the number of sensor nodes $\mathrm{N}$ is fixed, with the increase of the roadway length, the comparison of energy consumption for OD1 and OD4 is as shown in Fig. (9).

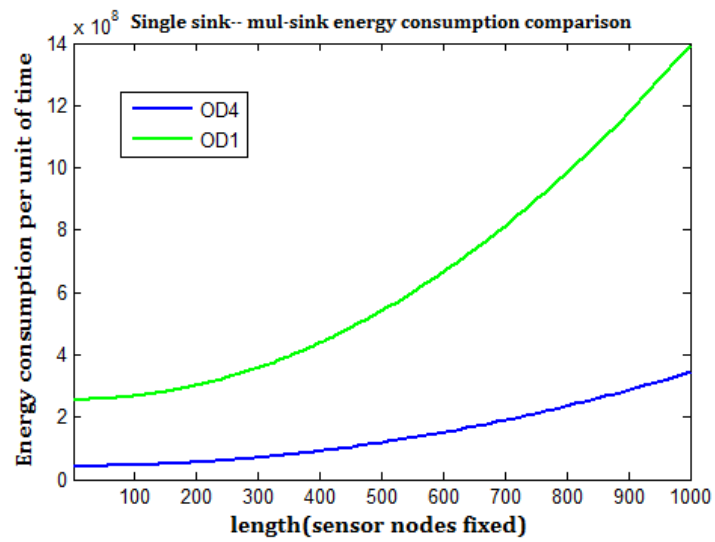

Fig. (9). Comparison of energy consumption for OD1 and OD4 with a fixed $\mathrm{N}$. 
From the above figure, with the increase of the roadway length, the energy consumption for two deployments is continuously increased, and the rate of increase for OD4 is smaller.

\section{CONCLUSION}

There are a lot of research work on WSN node deployment, but there are still few studies on the WSN node deployment in special and complex geographical environment such as the mine roadway. On the basis of linear WSN study, this paper proposes a WSN sink node optimization deployment in the middle suitable for the mine roadway, and provides the calculation method of optimized distance between nodes. Compared with the existing node uniform deployment and sink at one end, this model not only solves the problem of "energy hole" but also greatly reduces the total energy consumption of the system and the single node, and prolongs the network life cycle. This paper further proposes mul-sink nodes optimization deployment scheme, and determine the optimized number of sinks to minimize the total energy consumption of the system in consideration of the constraint of system equipment cost. The experimental simulation proves that two deployment schemes proposed in this paper optimize the energy consumption of network and significantly prolong the network life cycle. However, the energy consumption for too many nodes caused by overlapping coverage of the node monitoring areas is ignored, so it is the research direction for next step about how to solve this problem.

\section{CONFLICT OF INTEREST}

The authors confirm that this article content has no conflict of interest.

\section{ACKNOWLEDGEMENTS}

The project was supported by National Natural Science Foundation of China (61503316) and Major scientific and technological projects of Fujian Province China (2011H6027).

\section{REFERENCES}

[1] Z. Wang, Research of Key Technology of Wireless Sensor Network Deployment Strategies for Mine Complex Environment, M.S. thesis, Chinese University of Ming \& Technology, 2013.

[2] L. Yang, "Study of Coal Mine Gas Monitoring Wireless Sensor Network", M.S. thesis, Chinese University of Mining and Technology, 2008.

[3] I.F. Akyildiz, S. Weilian, and Y. Sankarasubramaniam, "A survey on sensor networks", IEEE Communications Magazine, vol. 40, no. 8 , pp. 102-114, 2002.

[4] Z. Wang, X. Zhao, and X. Qian, "Analysis of a scheduling strategy with energy balance for linear wireless sensor networks", Journal of University of Science and Technology of China, vol. 42, no. 6 , pp. 467-474, 2012.

[5] K. Joshua, and S. M. Loo, "A unified wireless sensor network framework”, SysCon 2012 - 2012 IEEE International Systems Conference, Proceedings, 2012, pp. 69-74.

[6] Y. Zhang, and Z. Wei, "On deployment optimization strategy for hybrid wireless sensor networks", In: $26^{\text {th }}$ Chinese Control and Decision Conference, CCDC, 2014, pp. 1875-1880.

[7] K. Lu, and Y. Liu, "Deploying nodes scheme in line wireless sensor networks," Computer Applications, vol. 27, no. 7, pp. 15661568, 2007.

[8] F. Tian, C. Wang, and W. Guo, "Linear clustering algorithm by competitive learning suitable for coal mine in wireless sensor networks", International Journal of Distributed Sensor Networks, vol. 5, no. 1, pp. 60, 2009.

[9] H. Ma, and J. Dang, "A linear clustering topology based on wireless sensor networks," Transducer and Microsystem Technologies, vol. 29, no. 8, pp. 48-51, 2010.

[10] L. Yu, and X. Yan, "A deployment method for long distance chaintype wireless sensor network", Microcomputer Applications vol. 30, no.5, pp.18-20, 2014.

[11] R. Zhang, "Research on Key Technologies of Wireless Sensor Network Based on Energy Efficiency", M.S. Thesis, Shandong University, 2007.

[12] K. Rakhi, A. Kapur, and M.D. Chawhan, "Energy efficient wireless sensor network", In: Proceedings - International Conference on Electronic Systems, Signal Processing, and Computing Technologies, ICESC, 2014, pp. 29-33.

[13] M. E. Keskin, "Wireless sensor network lifetime maximization by optimal sensor deployment, activity scheduling, data routing and sink mobility", Ad Hoc Networks, vol. 17, pp. 18-36, 2014.

[14] K. Vibhav, "Optimal nonuniform deployment of sensors for distributed detection in wireless sensor networks", $A C M$ Transactions on Sensor Networks, vol. 10, no. 2, Article No. 29, 2014.

[15] M. Keshavarz, and M. D. T. Fooladi, "Extended lifetime routing in wireless sensor network", In: Proceedings - $20135^{\text {th }}$ International Conference on Geo-Information Technologies for Natural Disaster Management, GiT4NDM, 2014, pp. 6-41. 